\title{
CHARACTERIZATION OF THE RESPONSE MAPS OF ALTERNATING-CURRENT NETWORKS*
}

\author{
GÜNTER ROTE
}

\begin{abstract}
In an alternating-current network, each edge has a complex conductance with positive real part. The response map is the linear map from the vector of voltages at a subset of boundary nodes to the vector of currents flowing into the network through these nodes. In this paper, it is proved that the known necessary conditions for a linear map to be a response map are sufficient, and we show how to construct an appropriate network for a given response map.
\end{abstract}

Key words. Alternating current, Electrical network, Dirichlet-to-Neumann map.

AMS subject classifications. 34B45, 94C05.

1. Problem statement and background. An alternating-current network is an undirected graph $G$ in which each edge $u w$ is assigned a conductance $c_{u w}=c_{w u} \in \mathbb{C}$ with positive real part: $\operatorname{Re} c_{u w}>0$. Such networks can model the physics of alternating current with a fixed frequency in an electrical network of conductors, capacitors, and inductors [5, Section 2.4]. At least 2 of the nodes are designated as boundary nodes (or terminals). Remaining nodes are called interior nodes.

A voltage is a complex-valued function $V_{u}$ on the set of nodes such that the equilibrium condition

$$
\sum_{u w} c_{u w}\left(V_{u}-V_{w}\right)=0
$$

holds for each interior node $u$, where the sum is taken over the edges $u w$ incident to $u$. In a connected network, the voltage is uniquely determined by its boundary values [5, Section 5.1]. The current flowing into the network through a boundary node $u$ is

$$
I_{u}:=\sum_{u w} c_{u w}\left(V_{u}-V_{w}\right)
$$

The response map is the linear map that takes the vector $\left(V_{u}\right)$ of voltages at the boundary nodes to the vector $\left(I_{u}\right)$ of currents flowing into the network through the boundary nodes.

Which linear maps are response maps of alternating-current networks? This question has been posed as an open problem [5, Problem 4.8], see also [6, Questions 1 and 2]. This note settles the problem: Theorem 2.1 shows that the known necessary conditions are sufficient.

Prasolov and Skopenkov [5] investigated alternating-current networks in connection with tilings of squares (or more general polygonal shapes) by rectangles. The prototypical problem in this area is to decide whether a square can be tiled by rectangles whose aspect ratio is selected from a range of given values $c_{1}, \ldots, c_{n}$, see [5, Problem 1.2]. (The aspect ratio of a rectangle is the height divided by the width.) The rectangles can have arbitrary size, and the number of rectangles of each aspect ratio $c_{i}$ is not fixed. For the

\footnotetext{
*Received by the editors on April 8, 2019. Accepted for publication on July 28, 2020. Handling Editor: Bryan L. Shader.

${ }^{\dagger}$ Freie Universität Berlin, Institut für Informatik, Takustr. 9, 14195 Berlin, Germany (rote@inf.fu-berlin.de).
} 
Electronic Journal of Linear Algebra, ISSN 1081-3810

A publication of the International Linear Algebra Society

Volume 36, pp. 698-703, October 2020 .

case of two values $c$ and $1 / c$, (in other words, for one shape which may be scaled and rotated), Prasolov and Skopenkov used alternating-current networks to give a new proof of the known characterization [5, Theorem 1.5]. For the general case, they derived some necessary conditions [5, Theorem 1.3], and they expressed hopes that the conjectured solution of their question (our Theorem 2.1) might allow further progress towards a full characterization [5, Section 4.2].

The general electrical impedance tomography problem is to reconstruct the network from its response map. This problem is more difficult and can only be solved when the structure of the network is constrained, cf. $[1,2,3,4]$.

\section{Statement and discussion of the characterization.}

TheOREM 2.1. Let $\Lambda=S+$ Ti be $a b \times b$ complex symmetric matrix with $S$ and $T$ real, for $b \geq 2$. Then $\Lambda$ describes the response map of some connected alternating-current network $G$ with boundary nodes if and only if it satisfies the following conditions:

1. $\Lambda$ has row sums 0 .

2. The real part $S$ is positive semidefinite.

3. The only solutions of $S x=0$ are the constant vectors $x=(c, c, \ldots, c)^{T}$.

If $\Lambda$ is given, one can construct a suitable network $G$ with $2 b-2$ nodes.

It has been shown by Prasolov and Skopenkov that these conditions on $\Lambda$ are necessary, see in particular [5, Lemma 5.2 (5)] for condition 2 and [5, Remark 5.3] for condition 3, which depends on $G$ being connected. For the more familiar direct-current networks, i. e., networks with real (and nonnegative) conductances, it is known that the response matrix must fulfill the above conditions $1-3$, along with the condition that the off-diagonal elements are $\leq 0$. In this case, sufficiency is trivial, since one can take $\Lambda$ directly as the Laplace matrix (see Section 3 for the definition) of a network, without any interior nodes.

For alternating-current networks, sufficiency of conditions 1-3 is easy for $b=2$, by the same reason as for direct-current networks: Condition 1 implies that $\Lambda$ is of the form $\left(\begin{array}{cc}c & -c \\ -c & c\end{array}\right)$, and by conditions 2 and 3 , $c$ must have positive real part. No interior nodes are needed: the network consists of a single edge of conductance $c$. For $b \geq 3$, however, the matrix $\Lambda$ can have off-diagonal entries with positive real part, and this implies that interior nodes are required, as discussed in Section 5 for the example of the $3 \times 3$ matrix (5.6). For $b=3$, sufficiency has been established by Prasolov and Skopenkov [5, Theorem 4.7], using one interior node. Their construction is different from ours when specialized to the case $b=3$. We do not know whether the number $b-2$ of interior nodes is optimal for $b \geq 4$.

3. The Laplace matrix and the response matrix. We will now recall how the matrix of the response map is computed, and we will prove a simple lemma that will be useful. The statements of this section are basic linear algebra and hold both over the reals and over the complex numbers.

In the rest of the paper, $I_{n \times n}$ denotes the $n \times n$ unit matrix, $J_{m \times n}$ denotes the all-ones matrix of dimension $m \times n$, and $j_{n}=J_{n \times 1}$ denotes the all-ones column vector of size $n$.

We can assume without loss of generality that the network has no loops: $c_{u u}=0$. The Laplace matrix (or Kirchhoff matrix) L of the network is a symmetric matrix, which is defined as follows: The off-diagonal 
edges $L_{u v}$ for $u \neq v$ are the negative conductances:

$$
L_{u v}= \begin{cases}-c_{u v}, & \text { if there is an edge between } u \text { and } v \\ 0, & \text { otherwise. }\end{cases}
$$

The diagonal elements $L_{u u}$ are chosen to make the row sums 0:

$$
L_{u u}=\sum_{u w} c_{u w}
$$

If there are no interior nodes, the response matrix is equal to $L$. Otherwise, the response matrix can calculated from $L$ as follows. Assume that the nodes $1,2, \ldots, b$ are the boundary nodes, and $b+1, \ldots, b+n$ are the interior nodes. Partition $L$ into blocks accordingly:

$$
L=\left(\begin{array}{cc}
A & B \\
B^{T} & C
\end{array}\right)
$$

with $A \in \mathbb{C}^{b \times b}, B \in \mathbb{C}^{b \times n}$, and $C \in \mathbb{C}^{n \times n}$.

Proposition 3.1. Let $L$ be the Laplace matrix of a connected network $G$ with at least one interior node, partitioned into blocks according to (3.3). Then the submatrix $C$ is invertible, and the response matrix $R$ is equal to the Schur complement of $C$ in $L$ :

$$
R=A-B C^{-1} B^{T}
$$

This well-known formula follows easily from writing the equations (1.1-1.2) in block form and substituting the solutions, see [3, Theorem 2.3] or [4, Lemma 3.8 and Theorem 3.9].

Lemma 3.2. Assume that $L$ is $a(b+n) \times(b+n)$ matrix of the form $(3.3)$, $C$ is invertible, and the last $n$ row sums of $L$ are zero. Then the row sums of the response matrix $R=A-B C^{-1} B^{T}$ are zero if and only if the first $b$ row sums of $L$ are zero.

Proof. By assumption, the last $n$ row sums of $L$ are zero: $B^{T} j_{b}+C j_{n}=0$, which implies $C^{-1} B^{T} j_{b}=-j_{n}$. In view of this, zero row sums of $R$ mean that $0=R j_{b}=A j_{b}-B C^{-1} B^{T} j_{b}=A j_{b}+B j_{n}$, which in turn expresses the fact that the first $b$ row sums of $L$ are zero.

4. Proof of sufficiency and construction of the network. Before giving the proof, we will study the simple example of just one interior node $y$ and boundary nodes $x_{1}, \ldots, x_{b}$, see Figure 1 . We give the edge

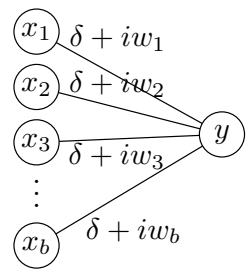

Figure 1. A network with one interior node $y$.

between $x_{u}$ and $y$ a conductance $\delta+i w_{u}$ with a small positive real part $\delta$, leaving the imaginary part $w_{u}$ as 
Electronic Journal of Linear Algebra, ISSN 1081-3810

a parameter, subject to the constraint $\sum_{u=1}^{b} w_{u}=0$. Calculating the response matrix $R$ by Proposition 3.1 gives $\operatorname{Re} r_{u v}=\left(w_{u} w_{v}-\delta^{2}\right) / \delta b$ for the off-diagonal entries. Thus, with this method, one can produce, for the real part of the response matrix, any positive semidefinite rank-one matrix $\left(w_{u} w_{v}\right) / \delta b$ with row sums 0 , up to a small error $\delta / b$ in all entries.

By inserting more interior nodes in this way, we can build up a sum of positive semidefinite rank-one matrices, and hence an arbitrary positive semidefinite matrix $S$ with row sums 0 . This is the main idea of the construction for the real part $S$ of $\Lambda$. We must take care of the accumulated error terms in the entries. We are able to accommodate them since is there is some tolerance for changing all off-diagonal entries of $S$ by the same amount while keeping the eigenvalues nonnegative. We will in fact choose the parameter $\delta$ in such a way such that $S$ gains an additional zero eigenvalue, and this will allow us to save one interior node in the construction.

The complex part of $\Lambda$ can be handled as an afterthought. We assign a fixed positive real conductance to every edge between two boundary nodes. This gives us the freedom to adjust the complex part of these edges as we like. In this way, we can achieve any desired complex part of the response matrix.

We now begin with the formal proof of Theorem 2.1. As mentioned in Section 2, the case $b=2$ can be easily handled without interior nodes. We thus assume $b \geq 3$ in order to avoid degenerate situations. Since the real part $S$ of the desired response matrix is symmetric, it can be written as

$$
S=U D U^{T}
$$

with a diagonal matrix $D=\operatorname{diag}\left(\lambda_{1}, \ldots, \lambda_{b}\right)$ whose entries are the eigenvalues

$$
\lambda_{1} \leq \lambda_{2} \leq \cdots \leq \lambda_{b}
$$

and an orthogonal matrix $U$ whose columns are the corresponding normalized eigenvectors of $S$. Since $S$ is positive semidefinite, all eigenvalues are nonnegative. By assumption $3, S$ has only one zero eigenvalue: $0=\lambda_{1}<\lambda_{2}$. From assumption 3 (or 1 ) of the theorem, we know the eigenvectors corresponding to $\lambda_{1}=0$ : they are the multiples of $j_{b}$. Thus, we can take the vector $j_{b} / \sqrt{b}$ as the first column of $U$.

We now decrease all positive eigenvectors by $\lambda_{2}$, so that they remain nonnegative. Algebraically, we replace the diagonal matrix of eigenvalues $D$ by

$$
D^{\prime}=D-\lambda_{2}\left[I_{b \times b}-\left(\begin{array}{cccc}
1 & 0 & 0 & \cdots \\
0 & 0 & 0 & \cdots \\
0 & 0 & 0 & \cdots \\
\vdots & \vdots & \vdots & \ddots
\end{array}\right)\right]
$$

and this results in the matrix

$$
S^{\prime}=U D^{\prime} U^{T}=U D U^{T}-\lambda_{2} U U^{T}+\lambda_{2} j_{b} / \sqrt{b} \cdot j_{b}^{T} / \sqrt{b}=S-\lambda_{2} I_{b \times b}+\lambda_{2} J_{b \times b} / b .
$$

In other words, $S^{\prime}$ is obtained from $S$ by increasing each off-diagonal entry by $\lambda_{2} / b$ and adjusting the diagonal so that the row sums remain 0 .

It will be convenient to rewrite (4.4) in a different way:

$$
S^{\prime}=U \sqrt{D^{\prime}} \sqrt{D^{\prime}} U^{T}=\left(U \sqrt{D^{\prime}}\right)\left(U \sqrt{D^{\prime}}\right)^{T}=V V^{T}
$$

where the columns of $V=U \sqrt{D^{\prime}}$ are no longer normalized. The columns of $V$ correspond to the interior nodes that we will add to the network. We can reduce their number by observing that, as the first two 
diagonal entries of $D^{\prime}$ are zero, the first two columns of $V$ are zero. They contribute nothing to $S^{\prime}$ and can be omitted, resulting in the real $b \times(b-2)$ matrix $W$ with

$$
W W^{T}=S^{\prime}=S-\lambda_{2} I_{b \times b}+\lambda_{2} J_{b \times b} / b .
$$

To obey the conventions of Section 3, we denote by $n=b-2$ the number of columns of $W$. (If the eigenvalue $\lambda_{2}$ has higher multiplicity, then more columns of $V$ are zero and $n$ can be further reduced.) Since the columns of $U$ are orthogonal and its first column is $j_{b} / \sqrt{b}$, the remaining columns of $U$, and hence, all columns of $W$ are orthogonal to $j_{b}$ :

$$
W^{T} j_{b}=0 .
$$

We are now ready to define the network. The imaginary parts of the conductances of the edges between the boundary nodes are represented by a symmetric real $b \times b$ matrix $F$ that will be determined later. With the parameters $\delta:=\lambda_{2} / 2 n$ and $\varepsilon:=\sqrt{b \delta}$, we set up the symmetric $(b+n) \times(b+n)$ matrix

$$
L:=\left(\begin{array}{cc}
\lambda_{2} I_{b \times b}-\frac{\lambda_{2}}{2 b} \cdot J_{b \times b}+i F & -\delta J_{b \times n}+\varepsilon i W \\
-\delta J_{n \times b}+\varepsilon i W^{T} & \delta b I_{n \times n}
\end{array}\right) .
$$

We have to show that it yields the desired response matrix $\Lambda$, and that it is indeed the Laplace matrix of a network with $n$ interior nodes. Let us calculate the response matrix $R$ by Proposition 3.1:

$$
R=\lambda_{2} I_{b \times b}-\frac{\lambda_{2}}{2 b} \cdot J_{b \times b}+i F-\left(-\delta J_{b \times n}+\varepsilon i W\right)\left(\delta b I_{n \times n}\right)^{-1}\left(-\delta J_{n \times b}+\varepsilon i W^{T}\right)
$$

Its real part is

$$
\begin{aligned}
\operatorname{Re} R & =\lambda_{2} I_{b \times b}-\lambda_{2} / 2 b \cdot J_{b \times b}-\frac{1}{\delta b}\left(\delta^{2} n J_{b \times b}-\varepsilon^{2} W W^{T}\right) \\
& =\lambda_{2} I_{b \times b}-J_{b \times b}\left(\lambda_{2} / 2 b+\delta n / b\right)+W W^{T} \\
& =\lambda_{2} I_{b \times b}-J_{b \times b}\left(\lambda_{2} / 2 b+\lambda_{2} / 2 b\right)+S-\lambda_{2} I_{b \times b}+J_{b \times b} \cdot \lambda_{2} / b=S,
\end{aligned}
$$

as desired. Since we can choose $F$ arbitrarily, the imaginary part of $R$ can be adjusted to any desired value $T$. The straightforward calculation gives the explicit formula

$$
F:=T-\sqrt{\delta / b}\left(W J_{n \times b}+J_{b \times n} W^{T}\right) .
$$

Thus, we have achieved $R=\Lambda$.

To conclude the proof, we still have to show that $L$ is the Laplace matrix of a network whose conductances have positive real parts: (a) All off-diagonal elements of $L$, whenever they are nonzero, have negative real parts, namely $-\lambda_{2} / 2 b$ or $-\delta$, and hence, the corresponding conductances have positive real parts. (b) Finally, we need to check that the row sums of $L$ are zero. The sums of the last $n$ rows are $-\delta J_{n \times b} j_{b}+\varepsilon i W^{T} j_{b}+$ $\delta b I_{n \times n} j_{n}=-\delta b j_{n}+0+\delta b j_{n}=0$, by applying (4.5) for the second term. Since the row sums of $R=\Lambda$ are 0 by assumption, Lemma 3.2 allows us to conclude without further calculation that the first $b$ row sums of $L$ are also 0 .

5. An example. We have seen that the imaginary part of $\Lambda$ is not an issue. Thus, for simplicity, we choose a real matrix as an example:

$$
\Lambda=\left(\begin{array}{rrr}
2 & 1 & -3 \\
1 & 2 & -3 \\
-3 & -3 & 6
\end{array}\right)
$$


This matrix has some positive off-diagonal entries. Hence, it is not the response matrix of a network without interior nodes, and it cannot be the response matrix of any direct-current network whatsoever.

The eigenvalues of $\Lambda$ are $\lambda_{1}=0, \lambda_{2}=1, \lambda_{3}=9$. The matrix $W$ has $n=1$ column, which is the properly scaled eigenvector $\sqrt{\lambda_{3}-\lambda_{2}} \cdot(1,1,-2)^{T} / \sqrt{6}$ corresponding to $\lambda_{3}$. One can recognize this vector in the last column of the matrix $L$ below in the imaginary parts. Our method sets $\delta=1 / 2, \varepsilon=\sqrt{3 / 2}$, and constructs the following Laplace matrix:

$$
L=\left(\begin{array}{ccc|c}
+\frac{5}{6}-\frac{2}{3} i \sqrt{2} & -\frac{1}{6}-\frac{2}{3} i \sqrt{2} & -\frac{1}{6}+\frac{1}{3} i \sqrt{2} & -\frac{1}{2}+i \sqrt{2} \\
-\frac{1}{6}-\frac{2}{3} i \sqrt{2} & +\frac{5}{6}-\frac{2}{3} i \sqrt{2} & -\frac{1}{6}+\frac{1}{3} i \sqrt{2} & -\frac{1}{2}+i \sqrt{2} \\
-\frac{1}{6}+\frac{1}{3} i \sqrt{2} & -\frac{1}{6}+\frac{1}{3} i \sqrt{2} & +\frac{5}{6}+\frac{4}{3} i \sqrt{2} & -\frac{1}{2}-2 i \sqrt{2} \\
\hline-\frac{1}{2}+i \sqrt{2} & -\frac{1}{2}+i \sqrt{2} & -\frac{1}{2}-2 i \sqrt{2} & \frac{3}{2}
\end{array}\right) .
$$

\section{REFERENCES}

[1] Y. Colin de Verdiére. Réseaux électriques planaires I. Commentarii Mathematici Helvetici, 69:351-374, 1994.

[2] Y. Colin de Verdiére, I. Gitler, and D. Vertigan. Réseaux électriques planaires II. Commentarii Mathematici Helvetici, $71: 144-167,1996$.

[3] E.B. Curtis, D. Ingerman, and J.A. Morrow. Circular planar graphs and resistor networks. Linear Algebra and its Applications, 283:115-150, 1996.

[4] E.B. Curtis and J.A. Morrow. Inverse Problems for Electrical Networks. World Scientific, Singapore, 2000.

[5] M. Prasolov and M. Skopenkov. Tiling by rectangles and alternating current. Journal of Combinatorial Theory, Series A, 118:920-937, 2011.

[6] M. Skopenkov. Problem 3. Inverse problem for alternating-current networks. Open Problems in Discrete Differential Geometry, Oberwolfach Reports, European Mathematical Society Publishing House, 12(1):720-721, 2015. 